REVISTA DE SOCIOLOGÍA

$\mathrm{N}^{\mathrm{o}} 17-2003$

Facultad de Ciencias Sociales - Universidad de Chile

(P. 81-94)

\title{
Campo Y Sistema En La Teoría Sociológica. (Notas Sobre Una Convergencia)
}

\author{
Omar Aguilar Novoa*
}

Luego de la crisis de la teoría estructural-funcionalista, parecía que la sociología estallaba bajo la forma de varias teorías y paradigmas diferentes, cuya principal característica era el abandono de la pretensión de construir una teoría general de la sociedad. Sin embargo, durante los últimos decenios asistimos a un renacimiento de esta ambición teórica, e incluso a un renacimiento de la propia teoría parsoniana. ${ }^{56}$ Pero la situación no es similar a aquella del imperio del estructural-funcionalismo pues hoy en día nadie pretende identificar de buenas a primeras su propia teoría con la sociología. Al parecer entre los sociólogos existe la convicción de que la sociología es una ciencia multiparadigmática y que ello no impide su progreso, muy por el contrario, una de las condiciones de posibilidad del campo sociológico en tanto que parte del campo científico es la posibilidad de la confrontación y la discusión con armas científicas entre los diferentes investigadores y las diferentes posiciones al interior del campo sociológico. ${ }^{57}$

Es en este contexto que quisiéramos hacer algunos comentarios a propósito de la sociología de Pierre Bourdieu, quien fuera uno de los sociólogos que, desde nuestro punto de vista, no renució a la pretensión teórica de fundar una verdadera ciencia de la sociedad, incluso si el propio Bourdieu negara a menudo el carácter general de su empresa teórica. La pretensión teórica tal como la concebimos no tiene nada que ver sobre el carácter más o menos abstracto, más o menos general, más o menos empírico de la sociología. Se trata simplemente de la ambición de llegar a una comprensión de la sociedad como totalidad, como conjunto, donde incluso hay un lugar para la propia teoría que intenta comprenderla. Como lo veremos más adelante, este aspecto del conocimiento científico, en el que el observador se reencuentra en aquello que observa, es uno de los desafíos que se le presentan a la ciencia contemporánea.

Ahora bien, lo que nos interesa es comparar ciertos aspectos de la teoría del sociólogo del Collège de France con la teoría de sistemas autopoiéticos. Concretamente interesa comparar el concepto de campo que utiliza Bourdieu con el concepto de sistema autorreferencial de Luhmann. Luego procederemos a comparar el enfoque del sociólogo

\footnotetext{
* Profesor del Departamento de Sociología, Universidad de Chile.

${ }^{56}$ Especialmente en la obra de MUNCH, R. (1987) Theory of Action. Towards a New Synthesis Going Beyond Parsons, Routledge \& Kegan Paul, London.

${ }^{57}$ Cf. BOURDIEU, P. (1988), "Vive la crise! For heterodoxy in social science" en Theory and Society $17: 773-787$
} 
francés sobre la reflexividad, con aquel de Maturana y Varela sobre la observación de segundo orden.

\section{Autonomía del campo y autorreferencialidad del sistema.}

Uno de los postulados ontológicos de la teoría de Pierre Bourdieu es aquel sobre el carácter relacional de lo real. Como lo dice a menudo el propio Bourdieu, la sociedad puede ser concebida como un espacio diferenciado de posiciones sociales en el que las relaciones entre estas posiciones constituyen el núcleo de lo que es propiamente social. ${ }^{58}$ Así, el sociólogo puede reconstruir el espacio social diferenciado a partir de la distribución de ciertas propiedades entre los agentes, especialmente bajo la forma de diferentes especies de capital, con las cuales ellos participan de las luchas por la tranformación o la mantención de la estructura del sistema de posiciones al interior del espacio social.

Podemos apreciar ya en esto que la teoría de Bourdieu utiliza nociones tomadas en préstamo a la física para dar cuenta de lo que ocurre en la sociedad. Espacio, distancia, posición, estructura, son nociones que tienen como objetivo el permitir aprehender la lógica de lo social, lógica que sería (como el propio Bourdieu los sostiene) una topo-logía, es decir un orden que se inscribe en un espacio bajo la forma de una distribución de los agentes según sus diferentes recursos, los que les permiten ocupar ahí una determinada posición.

En el conjunto de distinciones con las que Bourdieu observa la sociedad, el concepto de campo es uno de los más importantes. Dicho concepto está tomado también de la física pues corresponde a la noción de campo magnético con que esta disciplina describe varios de los fenómenos en términos de relaciones de fuerza entre cuerpos en un espacio físico definido.

Con la generalización de este concepto al campo de la sociología, el sociólogo francés quiere aprehender la heterogeneidad que se encuentra en el espacio social de las sociedades modernas. Estas sociedades son diferenciadas según varios criterios, lo que da lugar a la autonomización de diversos "sub-espacios" sociales que tienen sus propias lógicas de funcionamiento y que no pueden ser explicados por lo que ocurre fuera de ellos mismos.

Esta idea de la sociología de Bourdieu es cercana a aquella de Luhmann a propósito de los sistemas autopoiéticos ${ }^{59}$. Para el sociólogo de Bielefeld la sociedad moderna es una sociedad funcionalmente diferenciada. Esto quiere decir que los sistemas altamente complejos tienden a reducir complejidad por medio de la introducción del mismo principio de diferenciación que los constituye, es decir: la diferencia sistema/entorno. De esta forma, los sistemas complejos pueden introducir esta diferenciación en ellos mismos como

\footnotetext{
${ }^{58}$ BOURDIEU, P. (1980), Le sens pratique Editions de minuit, Paris; BOURDIEU, P. (1984), "Espace social et genése de classes", en Actes de la recherche en sciences sociales, 52-53:pp.3-15, BOURDIEU, P. (1987), Choses dites, Les éditions de minuit, Paris; y BOURDIEU, P. (1994), Raisons pratiques. Sur la théorie de l'action, Eds. du Seuil, Paris

${ }^{59}$ LUHMANN, N. (1991) Sistemas Sociales. Lineamientos para una Teoría General. Alianza Editorial, México.
} 
estrategia de reducción de la complejidad. La diferenciación sistémica es entonces la operación recursiva de la distinción de base sistema/entorno al interior del propio sistema.

Una de las diferencias importantes entre Bourdieu y Luhmann lo constituye el hecho de que este último elabora una teoría de la evolución social, para lo cual se apoya en el concepto de diferenciación funcional. Según el principio de Goldweiser y su ley de las posibilidades limitadas, problemas estructurales específicos pueden solamente tener un número limitado de soluciones posibles. Es por ello que en el curso de la evolución social solamente un número limitado de formas funcionalmente equivalentes de diferenciación funcional fueron desarrolladas, pues todas las posibilidades de diferenciación son reducidas por la cadena de selecciones que las preceden y que limitan las selecciones futuras. ${ }^{60}$

Las sociedades modernas son sociedades funcionalmente diferenciadas, es decir sociedades en las que la diferenciación funcional ha tenido como resultado el surgimiento de sistemas parciales, con un alto grado de autonomía y entre los que no existe ningún sistema que tenga un predominio sobre los otros pues cada uno de ellos tiene una función específica, es decir, un problema para el que el sistema tiene una solución equivalente desde un punto de vista funcional.

Utilizando la teoría de sistemas de Maturana y Varela, Luhmann concibe los sistemas funcionalmente diferenciados como sistemas autopoiéticos, dicho de otro modo, sistemas cerrados al ambiente desde el punto de vista de su organización y cuyos componentes son producidos por una red cerrada de operaciones sistémicas al interior del sistema mismo.

Así, para Bourdieu y para Luhmann, la economía, la política, la educación, la religión, el arte, entre otros, son sub-universos sociales que gozan de una autonomía funcional, que tienen sus propias lógicas de operación; aunque en la teoría de Bourdieu no se encuentra ninguna teoría general sobre el modo de funcionamiento de los diferentes campos sociales ni ninguna teoría de la evolución social sobre la cual apoyarse. ${ }^{61}$

Si bien estos dos sociólogos están de acuerdo sobre el carácter autónomo de estos sub-universos sociales en las sociedades modernas, la manera de comprenderlos no es exactamente la misma en uno y en otro autor. El concepto de campo y el concepto de sistema forman parte de matrices de distinciones totalmente diferentes. El concepto de campo no puede ser aislado de los de capital, habitus, juego, estrategia, relaciones de fuerza, etc. ${ }^{62}$ Al mismo tiempo, el concepto de sistema en Luhmann no puede ser aislado de los de autorreferencia, autopoiesis, complejidad, entorno, contingencia, sentido, etc. ${ }^{63}$

A partir de esto se puede constatar que evidentemente hay diferencias notables entre ambas nociones. Sería demasiado fácil indicarlas pues las estrategias teóricas de Luhmann

\footnotetext{
${ }^{60}$ LUHMANN, N. (1982) The Differentiation of Society, Columbia University Press, New York

${ }^{61}$ Bourdieu declaró haber estado trabajando en un libro que expondría una teoría general sobre los campos, del que no sabemos si pudo ser terminado.

${ }^{62}$ Como lo dice el propio Bourdieu, este concepto es definido al interior del sistema teórico mismo. Cf. BOURDIEU, P. (1992), Réponses. Pour une anthropologie réflexive, Eds. du Seuil, Paris, pg. 71

${ }^{63}$ Es también el propio Luhmann quien lo dice. Cf. Luhmann (1991) op. cit. pg. 12
} 
y de Bourdieu son muy diferentes. En todo caso existen al menos tres aspectos a señalar como diferencias importantes entre lo que constituye un campo y lo que constituye un sistema.

En primer lugar, el problema de la naturaleza del campo y la del sistema. En Bourdieu el problema central es el de los principios de diferenciación que caracterizan a una sociedad determinada. Estos principios están a la base de la distribución de los agentes sociales en un espacio constituido de posiciones dieferenciadas y de las relaciones entre ellas. Un campo es, como lo hemos dicho ya, un micro-espacio social en el que existe una estructura de relaciones entre posiciones sociales. Es esto lo que está en juego en las luchas y relaciones de fuerzas entre los agentes que en él participan. Por el contrario, un sistema social es el resultado de la reducción de la complejidad del entorno. El sistema social es la solución al problema de la doble contingencia como condición de posibilidad de lo social. Como ya lo había señalado Parsons, toda interacción debe enfrentar el problema de que ninguna acción es posible si Alter hace depender sus actos de la acción de Ego, y si Ego pretende conectar su comportamiento con el de Alter. ${ }^{64}$ A diferencia de la solución vislumbrada por Parsons a este problema, Luhmann ve en el sentido una manera de reducir las posibilidades abiertas en una interacción y así hacer probable lo que de otro modo resulta muy improbable: cómo asegurar la conexión de eventos temporales que, como las acciones, tienen una muy corta duración. Un sistema social utiliza por lo tanto el sentido como estrategia de reducción de la complejidad, o dicho de otra forma, de selección de posibilidades. Para mantener la diferencia con su entorno el sistema debe aumentar su propia complejidad de cara a la del entorno. Así, la diferencia sistema/entorno es una diferencia de grados de complejidad y siempre está en la base de la formación de sistemas el problema de la complejidad del mundo. ${ }^{65}$

Otro aspecto que marca la diferencia entre estos dos conceptos es aquel de los componentes de un campo y los componentes de un sistema. Si suponemos que el campo y el sistema son ambos entidades que se pueden distinguir en la realidad y que constituyen una unidad, nos podríamos preguntar por los elementos de tales unidades.

En el caso del campo, los elementos que forman parte de él son principalmente posiciones interdependientes. Estas son ocupadas por los agentes (ya sea un individuo o una institución) que toman parte en las luchas por el cambio de la estructura del campo y de sus reglas.

Se debe hacer notar aquí, a propósito de este aspecto de la noción de campo, que cuando Bourdieu compara tal noción con la de sistema, el sociólogo del Collège de France niega que el campo tenga componentes, partes, pues según él un campo no es

\footnotetext{
${ }^{64}$ PARSONS, T. (1951) Towards a General Theory of Action, Harper and Row, New York

${ }^{65}$ En la célebre discusión entre Luhmann y Habermas, este último señala que esta idea luhmanniana sobre la complejidad como catalizador de sistemas es insostenible por cuanto, si se lleva la argumentación hasta el absurdo, habría que imaginar un mundo complejo que constituye un problema para nadie, un problema antes incluso que algo exista. Según Habermas, no puede existir complejidad sin estructuras sistémicas, de ahí el equívoco del postulado ontológico de Luhmann. Cf. HABERMAS, J. (1990) La lógica de las ciencias sociales, Tecnos, 1990, Madrid, pg. 316
} 
descomponible. $^{66}$ Desde nuestro punto de vista, Bourdieu confunde el concepto de elemento con el de componente. Si decimos que el campo está constituido por ciertos elementos esto quiere decir que lo que define el campo como tal es la interdependencia de sus elementos, sin los cuales el campo no sería un campo social. Es verdad que si un campo es dividido, lo que resulta de tal división no es reducible a la lógica del campo original y que este último no es una unidad definida por los sub-campos que resultaron de su división.

Por el contrario, los elementos constitutivos de un sistema social son comunicaciones. Un sistema social según Luhmann es un sistema autorreferencial de comunicaciones. Esto significa que las selecciones de posibilidades actualizadas por el sistema son transmitidas mediante la comunicación. Esta actualiza posibilidades gracias a una síntesis de tres selecciones: la selección de un contenido o tema de la comunicación, la selección del acto de notificación, o como se dice a menudo: el acto comunicativo, y finalmente la selección que permite el rechazo o la aceptación de lo comunicado. ${ }^{67}$

De esta manera el sistema reproduce él mismo los elementos de los que se constituye, por medio de una red de procesos de producción de elementos que i) regeneran continuamente, por medio de sus transformaciones e interacciones, la red que los produce, y que ii) constituyen el sistema en tanto que unidad concreta en el espacio en el que existe. Esta recursividad que caracteriza la organización de los sistemas autopoiéticos permite la estabilización de la diferencia con el entorno por medio de la reproducción autopoiética de las selecciones que son hechas en el proceso comunicacional.

Bourdieu y Luhmann rompen con una vieja tradición en la teoría social, tradición según la cual los hombres eran considerados como los elementos constitutivos de la sociedad. Para ambos sociólogos, esta idea no es sino la expresión de la filosofía del sujeto que caracteriza el pensamiento moderno. Para el sociólogo francés, el individuo no es sino la emanación del campo y es este último el verdadero objeto de la sociología y no los individuos en cuanto tales. Para Luhmann los individuos son sistemas psíquicos que forman parte del ambiente de los sistemas sociales.

Sin embargo, Bourdieu y Luhmann reconocen que un campo y un sistema no pueden existir sin individuos. Según Bourdieu, para estudiar un campo hay que hacerlo por medio de la distribución de propiedades entre los individuos pues la información accesible está enlazada a ellos. ${ }^{68}$ Por su parte Luhmann había discutido bastante la distinción entre acción y comunicación a propósito de la definición de los sistemas sociales. Según él, las acciones no son los elementos constitutivos de los sistemas sociales pues éstas suponen ya la comunicación del sentido de la acción y de su definición. Sin embargo, y de manera similar a la de Bourdieu, el sociólogo de Bielefeld reconoce que si bien los elementos de un sistema social son las comunicaciones, estas últimas son atribuidas a acciones, son

\footnotetext{
${ }^{66}$ BOURDIEU, P. (1992) op. cit. pg. 80

${ }^{67}$ LUHMANN, N. (1981), "L'invraisemblance de la communication", en Revue international de sciences sociales, vol. XXXIII, 1: 137-147., LUHMANN, N. (1986) "The autopoiesis of social systems", en GEYER, F. \& VAN DER ZOUWEN, J. Sociocybernetics Paradoxes, SAGE, London. y LUHMANN. N. (1991) op. cit.

${ }^{68}$ BOURDIEU, P. (1992), op. cit.
} 
enlazadas a acciones. Así, la comunicación no puede ser observada de manera directa pero puede ser deducida a partir de su asignación a una acción. ${ }^{69}$

Como acabamos de ver, la vieja filosofía intelectualista de la que habla Bourdieu y el viejo pensamiento europeo del que habla Luhmann son de una u otra manera puestos en cuestión por una ciencia de la sociedad que destrona al "Hombre" del centro de las ciencias humanas. Es éste uno de los postulados más resistidos por los adversarios de los dos sociólogos.

Otro aspecto a considerar como diferencia entre los conceptos de campo y de sistema es aquel relativo a los límites de uno y otro. Para Bourdieu los límites de un campo social son muy variables de un caso al otro y no pueden ser determinados a priori por el investigador. Un criterio a considerar para definir estos límites es el de los efectos del campo. Retomando una analogía con la física, Bourdieu señala que el campo social produce efectos sobre los agentes que en él participan, de la misma manera que un campo gravitacional ejerce una atracción sobre los cuerpos celestes. Es decir, los límites del campo terminan allí donde terminan sus efectos, y los participantes de un campo determinado serán todos quienes sufren sus efectos. ${ }^{70}$

En Luhmann, por el contrario, los límites del sistema son límites de sentido. Ello significa que los sistemas sociales utilizan el sentido como estrategia para reducir la complejidad del mundo y que lo que pertenece al sistema es definido según este criterio. De esta forma, los límites del sistema no coinciden necesariamente con límites físicos.

Pese a estas diferencias bien claras entre el concepto de campo y el de sistema, existen aun algunas similitudes notables entre ambos enfoques, incluso si el propio Bourdieu niegue en varias ocasiones que las haya. Por ejemplo, según Bourdieu, a diferencia de lo que ocurre en un campo, la dinámica del sistema es el producto de una suerte de "autodesarrollo inmanente de la estructura" en tanto que la dinámica del campo es el resultado de los conflictos internos al campo mismo. ${ }^{71}$ A nuestro juicio, Bourdieu no ha comprendido bien la noción de sistema autorreferencial pues de hecho, tanto el sistema como el campo son ambos determinados por su estructura.

Si todo lo que ocurre al interior de un sistema es determinado por la estructura del sistema mismo, se dice que se trata de un sistema estructuralmente determinado. Evidentemente que un sistema no existe sin un ambiente. Al contrario, el sistema y su ambiente participan de un acoplamiento estructural en el que cada uno de ellos gatilla cambios de estado en el otro. Lo que es importante de retener al respecto es que todo lo que ocurre en el sistema mismo (en términos de lo que es importante para él) es determinado por la estructura del propio sistema.

Esta idea no está tan alejada de la idea de Bourdieu sobre el funcionamiento del campo. Así por ejemplo, el sociólogo francés nos dice que "...la estructura del campo

\footnotetext{
${ }^{69}$ LUHMANN, N. (1986) op. cit.

${ }^{70}$ BOURDIEU, P. (1992) op. cit. Parte I, cap. 2

${ }^{71}$ BOURDIEU, P. (1992) op. cit. pg. 80
} 
económico determina todo lo que pasa en el campo (económico)..." ${ }^{72}$ Esta es la misma idea que expresa Luhmann cuando analiza el funcionamiento del sistema económico o el sistema político. Según él, la autorreferncialidad deviene la condición para todas las operaciones del sistema pues éste tiene necesidad de mantener la capacidad de producir y reproducir neuevos elementos por sí mismo. En este proceso de reproducción autopoiética, todo lo que es comunicación relevante para el sistema es producida por el propio sistema, sin ser determinado en esta operación por el ambiente. Si bien el sistema social está abierto a la información que viene del ambiente, ésta es transformada en comunicación relevante para el sistema al interior del sistema mismo. A propósito del sistema político por ejemplo, Luhmann señala que "...todo lo que tiene una relevancia política es producida por el sistema político mismo, gracias a la conexión con todo lo que ya tiene una relevancia política." ${ }^{73}$

Esta capacidad que tienen los sistemas autopoiéticos de transformar las informaciones que provienen del ambiente en comunicación relevante para el sistema gracias a la autopoiesis sistémica, les permite mantener un alto grado de autonomía en ambientes complejos. Un ejemplo de ello es dado por el propio Bourdieu a propósito del campo económico. Según él, "la denominada lucha política para modificar la estructura del campo económico es parte integrante del objeto de la ciencia económica." ${ }^{74}$

Un último alcance a propósito de este tema. Bourdieu afirma que la teoría de los campos, a diferencia de la teoría de los sistemas, concibe éstos como lugares de relaciones de fuerza y no solamente de sentido o de comunicaciones en el sentido de Luhmann. Sin embargo, se podría agregar que si bien los elementos que constituyen un campo y un sistema no son los mismos según uno y otro sociólogo, la concepción general del modo de funcionamiento de estos espacios sociales es más bien similar en lo que respecta al grado de autonomía que poseen. Es más, uno podría preguntarse si los conflictos al interior de un campo no son acaso definidos por el propio campo. En este sentido habría una suerte de autorreferencialidad del campo a nivel de sus elementos constitutivos y a nivel de sus operaciones fundamentales (autorreferencia basal). Dejaremos hasta aquí la comparación entre Bourdieu y Luhmann respecto a las nociones de campo y sistema. Veremos ahora cómo existe una convergencia entre la teoría del sociólogo del Collège de France y la de la epistemología naturalizada de Maturana y Varela. ${ }^{75}$

\section{La objetivación del sujeto objetivante y el problema del observador.}

En nuestra opinión las similitudes entre los trabajos de Bourdieu y los de Maturana y Varela son notables. En primer lugar, los dos enfoques representan una suerte de ruptura con lo que Echeverría llamó el paradigma de base de la modernidad ${ }^{76}$, especialmente con el

\footnotetext{
${ }^{72}$ BOURDIEU, P. (1987) op. cit. pg. 127 (en francés en el original)

${ }^{73}$ LUHMANN, N. (1990) Political Theory in the Welfare State, Walter de Gruyter, New York, pg. 41 (en inglés en el original)

${ }^{74}$ BOURDIEU, P. (1987) op. cit. pg. 127 (en francés en el original)

${ }^{75}$ El término "epistemología naturalizada" fue sugerido por varela, aun cuando ya había sido utilizado por QUINE, W. V. (1969) Ontological relativity and others essays, Columbia University Pres, New York.

${ }^{76}$ ECHEVERRIA, R. (1988), El búho de Minerva, PIIE, Santiago
} 
pensamiento cartesiano, que está fundado sobre una matriz dualista. Como es sabido, este paradigma de base de la modernidad se basa en la idea de autonomía de la razón y de la separación de sujeto y objeto. Según esta concepción dualista de la realidad, existe un sujeto transcendental que se enfrenta a un mundo de objetos. Un objeto es todo aquello que puede ser representado como dotado del atributo del ser. El sujeto tiene la capacidad de establecer una actitud objetivante hacia los objetos y de apropiarse de ellos. Según esto, el sujeto puede establecer dos tipos de relaciones con el mundo de objetos: puede representarse el mundo objetivo y puede actuar en él de una manera instrumental utilizando el conocimiento para controlarlo.

Bourdieu, Maturana y Varela ponen en duda este paradigma de la modernidad, aun cuando ellos no se definen a sí mismos como anti-modernistas o post-modernistas. A nuestro juicio, las dos teorías, intentando responder desde la ciencia al problema de las formas a priori de la experiencia subjetiva, llegan al abandono de la matriz dualista del paradigma moderno.

Así por ejemplo, Bourdieu supera la vieja dicotomía entre el subjetivismo y el objetivismo en sociología al interrogarse sobre las formas sociales a priori de la experiencia. Como lo señalaba ya en sus primeros trabajos, se pueden distinguir tres modos de conocimiento que se sitúan en diferentes niveles: i) la fenomenología, que concibe la experiencia primera como dada (allant de soi), ii) el objetivismo, que se pregunta por las condiciones de posibilidad de la experiencia primera, y iii) el enfoque praxeológico, que se pregunta por las condiciones de posibilidad de la pregunta sobre las condiciones de posibilidad de la experiencia primera. ${ }^{77}$

Esta formulación "bourdieurienne" afirma la necesidad de la objetivación del sujeto objetivante como la única posibilidad de asegurar la reflexividad en sociología, es decir, asegurar el conocimiento de los límites del conocimiento del mundo social.

Para Bourdieu el sujeto objetivante debería objetivarse él mismo, objetivando su propia posición al interior del campo de la producción cultural pues hay siempre un punto ciego, un "biais", como lo dice el propio Bourdieu, en la objetivación del mundo social. ${ }^{78}$

En nuestra opinión, esta idea del sociólogo francés es convergente con la teoría de Maturana y Varela sobre los sistemas observadores.

A partir de investigaciones en neurofisiología que datan de fines de los años cincuenta, se descubrió que no existe la posibilidad de que el sistema cognitivo pueda distinguir entre apariencia y realidad, pues se trataría de un sistema cerrado que posee clausura operacional. Es decir, el sistema no puede distinguir entre una realidad independiente y sus propias operaciones cognitivas. Esta diferencia es hecha por un observador que opera en un metadominio, y que al mismo tiempo opera con un sistema cognitivo que no puede distinguir una realidad independiente de sus propias operaciones en tanto que observador. Ese es el punto ciego de todo observador.

\footnotetext{
${ }^{77}$ BOURDIEU, P. (1972), Esquisse d'une théorie de la pratique, Droz, Geneve.

${ }^{78}$ BOURDIEU, P. (1992) op. cit.
} 
Como es sabido, estos descubrimientos han llevado a discutir los fundamentos de la ciencia moderna, especialmente aquellos que afirman que i) la realidad objetiva existe independientemente de lo que los observadores hacen o desean, aún cuando esta realidad no pueda ser totalmente conocida, y ii) que la validez de las afirmaciones y de las explicaciones científicas está fundada en la relación con tal realidad. ${ }^{79}$

Esta nueva epistemología naturalizada que se deriva de tales descubrimientos, señala que todos somos, en tanto investigadores, observadores que intentamos explicar lo que observamos. La paradoja es que nos encontramos nosotros mismos en una situación de observadores que observan, cuando llegamos a observar nuestra propia observación a fin de describir y de explicar lo que hacemos. En tanto que sistemas estructuralmente determinados, todo lo que pasa en nosotros ocurre como un cambio determinado en el curso de la dinámica interna, o gatillado (pero no determinado) por nuestras interacciones con el ambiente.

Lo que resulta importante de retener es el hecho de que las explicaciones científicas (como toda suerte de explicación, por lo demás) no pueden estar basadas sobre un ilusorio acceso privilegiado a lo real. Es más, las explicaciones científicas tienen que ver con las operaciones gracias a las que los observadores producen sus explicaciones y no tienen que ver con una realidad independiente de lo que los observadores hacen en tanto que observadores. Ello no quiere decir que la ciencia deba renunciar a la posibilidad de un conocimiento fiable del mundo. Al contrario, la epistemología naturalizada muestra cómo las explicaciones científicas no tienen necesidad de estar fundadas sobre el supuesto de una realidad objetiva, sin que ello signifique que no haya coherencias en el dominio de la operación de los sistemas vivos y sociales. Esta coherencia del operar de los sistemas es explicado por el acoplamiento estructural entre el sistema y su ambiente, distinción hecha por un observador .

El nuevo criterio de validación de las explicaciones científicas propuesto por la epistemología naturalizada pone el acento sobre el papel del observador en la generación del fenómeno a explicar.

Bourdieu, por su parte, pone en cuestión también los presupuestos inherentes a la posición de observador objetivo que asume el sociólogo, tal como ocurre en la tradición estructuralista, por ejemplo. Es por ello que afirma la necesidad de establecer una práctica reflexiva que permita objetivar al observador en su relación a los observadores observados. ${ }^{80}$ Si se analiza de manera atenta, se podrá ver cómo estos dos enfoques enfrentan el problema de la circularidad del conocimiento sin caer en el solipsismo estéril y sin retornar al dualismo cartesiano que hacía de la ciencia una suerte de torre de marfil en la que los investigadores se encontraban libres de toda determinación en su relación al objeto de conocimiento.

\footnotetext{
${ }^{79}$ MATURANA, H. (1990a) "Science and Daily Life: The Ontology of Scientific Explanations", en KROHN, W., KÜPPERS, G. \& NOWOTNY, H. [Eds.] (1990), Selforganization. Portrait of a Scientific Revolution, Kluwer Academic Publishers, Dordrecht/Boston/London.

${ }^{80}$ BOURDIEU, P. (1992) op. cit., al analizar algunos intentos de desarrollar una observación de segundo orden critica la categoría de observación que se encuentra en ciertos antropólogos americanos, pero desconoce la teoría de los sistemas observadores tal como ha sido desarrollada en Chile.
} 


\section{SOCIAL}

\section{CAMPO \\ CIENTÍFICO}
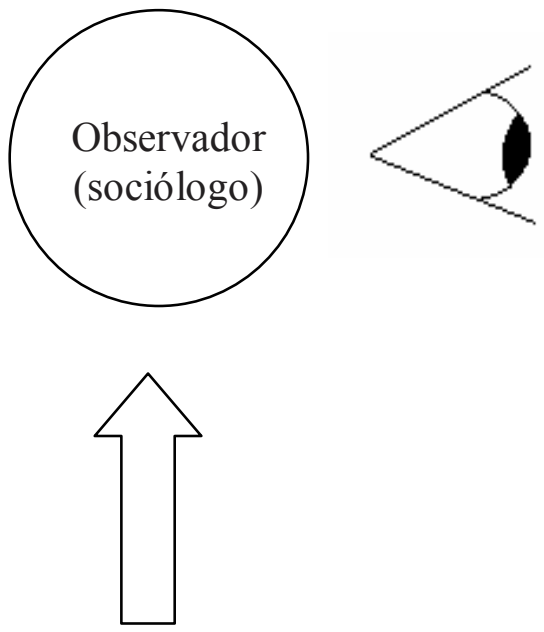
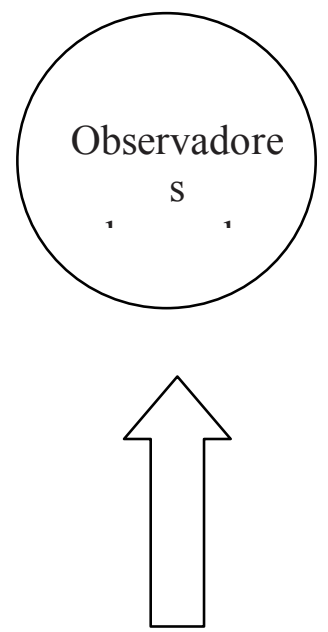

Condiciones sociales de producción

Condiciones sociales de producción

En la figura de arriba se puede ver cómo los postulados de Bourdieu y de la teoría de sistemas observadores resultan convergentes. El sociólogo es capaz, como observador, de objetivar el mundo social y ver el punto ciego de los observadores observados pero no puede ver, en la operación de observación misma, el propio.

Es más, los dos enfoques extraen también las consecuencias radicales de lo que descubren a nivel del proceso de conocimiento. Bourdieu, por ejemplo, afirma el carácter ilusorio de las dicotomías que caracterizan la discusión en el seno del campo sociológico (teoricistas/empiristas, objetivistas/subjetivistas, estructura/actor, etc.). Según el enfoque del sociólogo francés, estas dicotomías no tienen una base real sino social. Esto quiere decir que los sociólogos, en tanto que observadores de la sociedad (observadores de observadores, habría que agregar) no pueden escapar a las determinaciones que observan en el seno de los campos sociales. La sociología (y la ciencia en general) es un campo donde hay también fuerzas en lucha, intereses, posiciones diferenciadas, etc. Es por ello que el sociólogo no está libre de lo que él mismo observa afuera y el problema epistemológico consiste en la incapacidad que tiene el observador sociólogo de darse cuenta de este punto 
ciego. Dicho de otra manera, el observador observa el mundo social y es capaz de ver las condiciones sociales de producción de la experiencia primera en los observadores observados pero no es capaz de darse cuenta de las condiciones sociales de producción de su propia observación.

Como lo dice el propio Bourdieu, es este fundamento social lo que está a la base de muchas de las discusiones en términos de dicotomías en el seno del campo sociológico. Pensamos especialmente en la disputa estéril entre teoricistas y empiristas, o entre cuantitativistas y cualitativistas. A veces pareciera que cada término del dualismo pudiera ser explicado por el lugar que sus representantes ocupan en el sistema de posiciones al interior del campo sociológico y los diferentes juegos en los que participan.

Por otro lado, según Maturana, todo sistema racional, como por ejemplo una teoría científica, tiene un fundamento emocional. Dicho de otro modo, todo sistema racional tiene un fundamento irracional. ${ }^{81}$ Las emociones, en tanto que disposiciones corporales dinámicas, definen los diversos dominios de acción en los que nos movemos. En este sentido, todo sistema racional está constituido en un operar con premisas aceptadas a priori desde una cierta emoción. Así, el acuerdo o el desacuerdo al que lleguen dos o más individuos dependerá de que estos individuos acepten o no estas premisas. Maturana afirma que no se puede pretender una justificación trascendente de nuestro propio actuar diciendo: es racional. Bourdieu dirá que en sociología "una buena parte de los trabajos llamados de teoría o de metodología no son sino ideologías justificadoras de una forma particular de competencia científica". ${ }^{82}$ Esto quiere decir que tales disputas no tienen un fundamento racional. Maturana dirá que su fundamento es emocional en tanto que Bourdieu dirá que es social.

Así, Bourdieu y Maturana, desde posiciones diferentes en el campo científico, ponen entre paréntesis varias de las ideas que están a la base del pensamiento moderno, especialmente la idea de la autonomía de la razón y de la separación de sujeto y objeto. A nuestro juicio, el concepto de habitus como trascendental histórico y la idea de un estructuralismo constructivista y de un constructivismo estructuralistas son posibles gracias a este paradigma no cartesiano sobre el cual se apoya la teoría de Bourdieu.

Es cierto que hemos modificado un poco los términos utilizados por Bourdieu pero creemos que la epistemología naturalizada y la teoría de la reflexividad del sociólogo francés son postulados radicales sobre las posibilidades y los límites del conocimiento científico. Puede tratarse de enfoques complementarios. Habría que trabajar más la relación entre el fundamento emocional y el fundamento social al que ambos enfoques aluden.

Podríamos deducir que somos (en tanto que observadores) sistemas determinados en nuestra estructura biológica y social. Tenemos un punto ciego que se inscribe en el operar del sistema nervioso y al mismo tiempo tenemos un punto ciego que se inscribe en el campo social al que pertenecemos. Como lo decían Maturana y Varela, toda observación es una operación de distinción gracias a la cual distinguimos una unidad de su ambiente. Esta es la operación de base de los sistemas observadores y toda teoría es un conjunto de

\footnotetext{
${ }^{81}$ MATURANA, H. (1990b) Emociones y lenguaje en educación y política, Dolmen, Santiago.

${ }^{82}$ BOURDIEU, P. (1987) op. cit. pg. 87
} 
distinciones con las cuales operamos en el mundo al mismo tiempo que lo conocemos. "Todo hacer es conocer y todo conocer es hacer", según el aforismo de los investigadores chilenos.

Parafraseando a Pascal, quien decía que "el mundo me comprende y me engulle como un punto, pero yo lo comprendo", Bourdieu afirma que "el espacio social me engloba como un punto. Pero este punto es un punto de vista, el principio de una vista tomada a partir de un punto situado en el espacio social, de una perspectiva definida en su forma y su contenido por la posición objetiva a partir de la cual esta perspectiva es tomada." ${ }^{83}$

Pero nosotros, en tanto observadores, no podemos ver el punto ciego de nuestras propias distinciones. Como lo dice Bourdieu "cada sociólogo tiene interés en escuchar a sus adversarios en la medida misma que estos tienen interés en ver lo que él no ve, los límites de su visión, que por definición se le escapan." ${ }^{84}$ Es por ello que la autonomía del campo científico es uno de los principales desafíos hoy en día pues la ciencia requiere de un espacio social que le permita desarrollarse y definir por sus propios criterios lo que es científico de lo que no lo es.

${ }^{83}$ BOURDIEU, P. (1994) op. cit., pg. 28 (en francés en el original)

${ }^{84}$ BOURDIEU, P. (1987)op. cit., pg. 49 (en francés en el original) 